\title{
A Parameter Identification Method for Helicopter Noise Source Identification and Physics-Based Semi-Empirical Modeling
}

\author{
Eric Greenwood \\ NASA Langley Research Center
}

\author{
Fredric H. Schmitz \\ University of Maryland
}

\begin{abstract}
A new physics-based parameter identification method for rotor harmonic noise sources is developed using an acoustic inverse simulation technique. This new method allows for the identification of individual rotor harmonic noise sources and allows them to be characterized in terms of their individual non-dimensional governing parameters. This new method is applied to both wind tunnel measurements and ground noise measurements of two-bladed rotors. The method is shown to match the parametric trends of main rotor Blade-Vortex Interaction (BVI) noise, allowing accurate estimates of BVI noise to be made for operating conditions based on a small number of measurements taken at different operating conditions.
\end{abstract}

\section{Nomenclature}

$A$

$a$

$B$

$b_{1}$

$b_{2}$

$c$

$C_{T}$

$C_{v}$

$D$

$D_{0,}, D_{1 C}, D_{1 S}$

$e(t)$

$F(X)$

$M_{A T}$

$N_{o b s}$

$p$

$p^{\prime}(t)$

$p_{\text {meas }}^{\prime}$

$p_{X}^{\prime}$

$R$

$\mathcal{R}$

$r_{c}$

$r_{0}$

$r_{v}$

$s$

$T$

$T_{0}, T_{1 C}, T_{1 S}$

$t$

$u$

$u$

$v_{i}$

$w$

$X$

$x, y, z$

$x_{I}$ longitudinal inflow parameter

particle inertia weight

lateral inflow parameter

particle local weight

particle global weight

mean inflow parameter

thrust coefficient

vortex core growth rate

wake contraction parameter

drag loading harmonic coefficients

error between signals, $\mathrm{Pa}$

objective function

advancing tip Mach number

number of observers

power of generalized norm

acoustic pressure, $\mathrm{Pa}$

measured acoustic pressure, $\mathrm{Pa}$

simulated acoustic pressure, $\mathrm{Pa}$

rotor radius, $\mathrm{m}$

random variable

vortex viscous core radius, $m$

initial vortex core radius, $\mathrm{m}$

vortex "roll-up" radius, $\mathrm{m}$

wavelet scale

time of rotor period, $\mathbf{s}$

thrust loading harmonic coefficients

time, $\mathrm{s}$

wavelet domain signal

time domain signal

$i^{\text {th }}$ particle velocity

observer weight

design variables

hub-centered coordinates, $\mathrm{m}$

$\mathrm{i}^{\text {th }}$ particle position $\zeta$

wavelet basis function

vortex circulation strength, $\mathrm{m}^{2} / \mathrm{s}$

$\Gamma_{0} \quad$ mean vortex strength, $\mathrm{m}^{2} / \mathrm{s}$

$\gamma_{0}, \gamma_{1 C}, \gamma_{1 S} \quad$ vortex strength harmonic coefficients

$\lambda$ non-dimensional inflow (inflow/tip speed)

$\mu \quad$ advance ratio (airspeed/tip speed)

$\mu_{x} \quad$ horizontal advance ratio

$\mu_{z} \quad$ vertical advance ratio

$\sigma \quad$ wavelet shape parameter

$\phi \quad$ wake age

$\psi_{b} \quad$ blade azimuth

$\psi_{v} \quad$ vortex element azimuth

\section{Paper Objective}

It is the objective of the authors to develop a new physics-based and non-dimensional semi-empirical modeling method for the identification and quantification of impulsive helicopter noise sources using ground-based acoustic measurements.

\section{Background}

Measured helicopter noise data is widely used to generate helicopter source noise models that can be used in the prediction of ground noise contours for land use and mission planning purposes. The Rotorcraft Noise Model (RNM) is one empirical modeling method that is widely used by the Government and Industry in order to describe the noise radiation of rotorcraft. [1] Several similar empirical modeling methods are also under development.[2][3] However, current empirical models derived from ground-based measurements are developed dimensionally and cannot be rigorously extended to estimate noise radiation at other operating conditions, such as those at different gross weights, temperatures, or density-altitudes. In order to extend helicopter noise models to these variations, the nondimensional governing parameters of the dominant helicopter noise sources must be identified. For 
example, noise due to Blade-Vortex Interaction (BVI) is known to depend on several non-dimensional parameters such as the advance ratio $(\mu)$, thrust coefficient $\left(C_{T}\right)$, inflow ratio $(\lambda)$, and advancing tip Mach number $\left(M_{A T}\right)$. [4] In order to produce accurate noise predictions at conditions other than those for which the measurements were obtained, the noise produced by each source on the helicopter must be classified based on its non-dimensional governing parameters.

Existing methods of constructing source noise models from empirical data, such as RNM, often treat the helicopter as a single compact noise source. The noise radiated by this assumed source is described in terms of metrics like sound pressure level, $1 / 3$ octave, or narrowband frequency levels over the surface of a hemisphere set a fixed distance about the assumed source location. In reality, numerous distinct distributed sources contribute to helicopter external noise radiation, as illustrated in Figure 1. Each type of noise source has its own magnitude and directivity, which can vary in response to changes in flight condition, and may not be amenable to a compact source approximation. In order to accurately represent helicopter noise across complex flight trajectories composed of numerous flight conditions, these noise sources must first be described using the governing non-dimensional parameters and then individually identified and modeled.

In previous work by the authors, a novel technique to separate main rotor and tail rotor harmonic noise from ground-based measurements was developed.[6] However, the theory was limited by the assumption that all noise from the rotor radiated from a single point on the rotor hub. In reality, the actual rotor noise sources are distributed on or near the plane of the rotor. Although this assumption gave reasonable results for lower frequency noise sources, it introduced significant phase error for higher frequency sources, such as BVI. This theory also required on-board measurement of the main rotor revolutions to supply an accurate trigger for periodic averaging. In order to overcome these limitations and to successfully identify and characterize higher frequency noise sources using ground-based acoustic measurements, it is necessary to make use of additional physical knowledge of the rotor noise problem.

In this paper, a new parameter identification technique is introduced in order to separate the contributions of individual rotor noise sources from the measured signals, model these noise sources in terms of their governing non-dimensional parameters, and apply these models to make estimates of external noise radiation at operating conditions other than those at which noise was measured. BVI noise is emphasized in this paper; however, the technique is equally applicable to all other sources of harmonic rotor noise.

The parameter identification technique developed in this paper is applied to two datasets. The first dataset is the study of $a 1 / 7^{\text {th }}$ scale model of the $A H-1$

Operational Loads Survey rotor in the German-Dutch Wind Tunnel (DNW).[4] This dataset is notable for high quality acoustic measurements over a large range of operational conditions carefully controlled by the governing non-dimensional parameters of rotor harmonic noise sources. Using these data, the ability of the parameter identification technique to produce models correctly capturing the parametric variations of rotor noise is evaluated. The second dataset consists of ground noise measurements of the Bell 206B3 helicopter captured at Moffett Field, California, in 2006.[5] The ability of the parameter identification technique to build rotor noise models capable of accurately estimating external noise radiation over a wide range of flight conditions using a limited set of measured data is demonstrated.

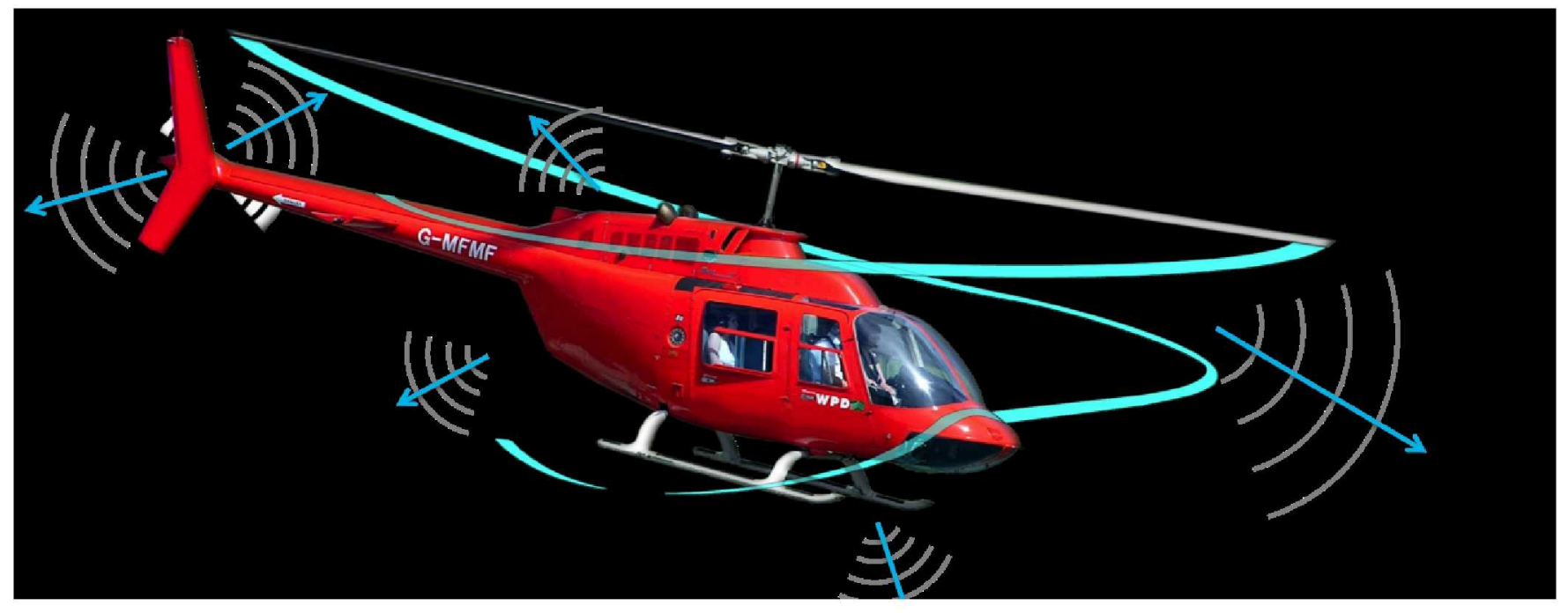




\section{Technical Approach}

A new semi-empirical method of noise estimation has been developed that is based upon a non-dimensional form of the governing helicopter noise relationships.

This method is illustrated in Figure 2. Rotor noise data measured across a wide range of flight conditions are first categorized in terms of the individual nondimensional governing parameters of each noise source. Then, the acoustic data are transformed into a common reference frame shared amongst all measured flight conditions. From these data, the main rotor (or tail rotor) harmonic noise is separated from all other noise sources on the helicopter. Finally, the separated rotor harmonic noise is used as an input to a parameter identification technique based on an assumed analytical model of rotor harmonic noise generation. When applied across a wide range of flight conditions, the result is a set of dependent physical modeling parameters associated with the nondimensional governing parameters of the rotor noise sources. By using these parameters with the associated analytical model, estimates of noise at flight conditions that are difficult to measure may be made.

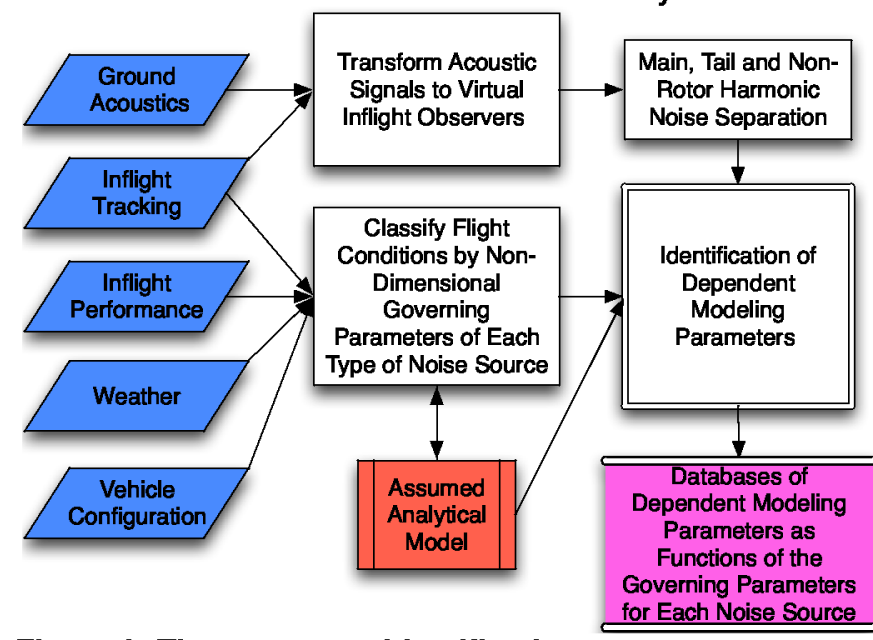

Figure 2: The parameter identification process.

Each non-dimensional governing parameter must have a corresponding range of acoustic measurements. For the main rotor harmonic noise sources modeled in this paper, the governing non-dimensional parameters used are rotor advance ratio, inflow ratio, thrust coefficient, and tip Mach number. For other noise sources, different non-dimensional governing parameters must be identified. The complete characterization of all typical flight conditions may require specialized testing and data gathering procedures to represent the operational flight conditions of interest.
Once the data has been classified, it must be transformed to a common reference frame shared by all acoustic measurements. A time domain deDopplerization technique [6] is applied to transform ground based noise measurements to those that would be measured by a set of "virtual inflight observers" traveling with the helicopter through the medium with approximately fixed measurement geometry - this is analogous to the noise measurement of a rotor in a wind tunnel.

After the acoustic signals are transformed to observers moving with the helicopter, the contribution of main rotor harmonic noise to the transformed acoustic signals may be identified using a periodic averaging technique. By applying the wavelet transform to the acoustic signals individual main rotor revolutions are identified, forming time windows for the averaging process, as shown in Figure 3. The wavelet transform is used to create an accurate time-frequency representation of the signal and is expressed with the convolution:

$$
U(s, t)=\int_{-\infty}^{\infty} u\left(t^{\prime}\right) \frac{1}{\sqrt{s}} \xi^{*}\left(\frac{t-t^{\prime}}{s}\right) d t^{\prime}
$$

where $s$ is the scale of the wavelet (related to the frequency), $t$ is the time of the transform, and $\xi^{*}$ is the complex conjugate of the wavelet basis function. The Ricker wavelet basis function is used to identify the time of each blade passage as it provides excellent temporal accuracy. This basis is expressed as:

$\zeta(x)=\frac{1}{\sqrt{2 \pi} \sigma^{3}}\left(1-\frac{x^{2}}{\sigma^{2}}\right) e^{\frac{-x^{2}}{2 \sigma^{2}}}$

where $\sigma$ is a shape parameter, typically chosen around unity. These bases are applied at scales associated with the frequencies between $90 \%$ and $110 \%$ of the nominal blade passage frequency. The power spectrum of the wavelet transform is calculated (middle of Figure 3), and a new signal composed from the highest power across all scales at each point in time (bottom). The peaks of this signal represent individual blade passages. The de-Dopplerized acoustic signal may now be divided into successive rotor periods (the red dashed lines in Figure 3 ) and then ensemble averaged to capture the main rotor periodic noise. 

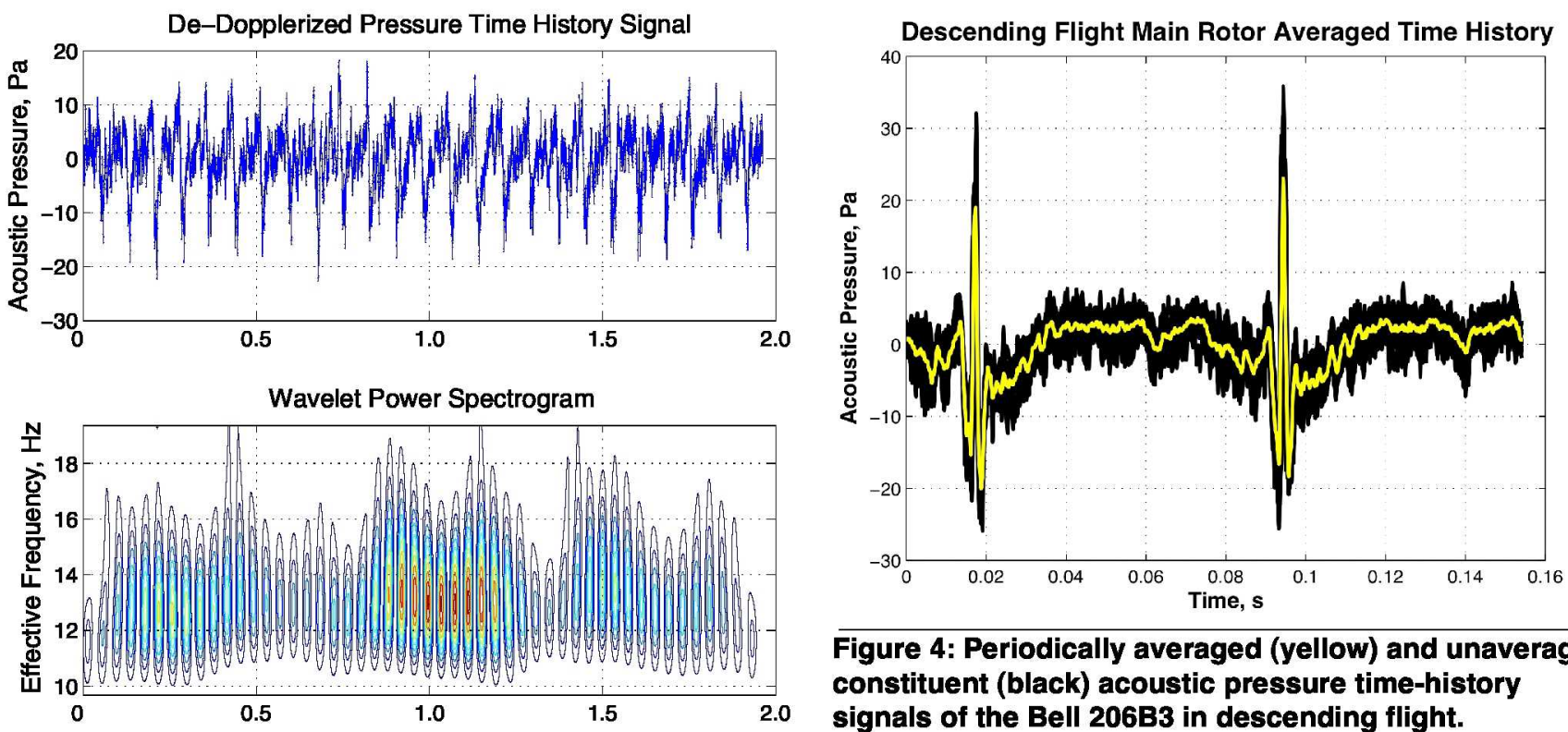

Figure 4: Periodically averaged (yellow) and unaveraged constituent (black) acoustic pressure time-history signals of the Bell 206B3 in descending flight.

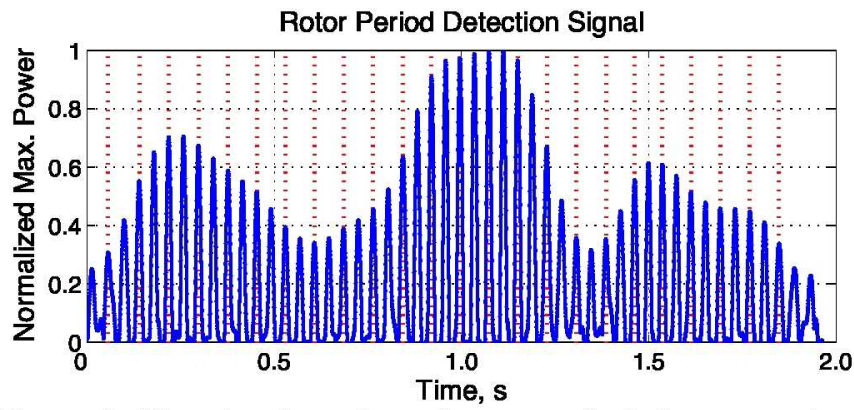

Figure 3: Wavelet detection of rotor periods for groundbased acoustic measurement of a Bell $206 \mathrm{B3}$.

The de-Dopplerization process does not accurately capture the phase of impulsive noise sources if they are distributed over a localized area of the rotor disk, such as BVI. A correction for this effect is developed by assuming that the impulsive helicopter noise is a point source, allowing the impulsive noise source to be captured in the averaged signal. The results are shown in Figure 4 for the Bell 206B3 helicopter in descending flight. Now having isolated the main rotor harmonic noise sources, they are identified and modeled in terms of their associated non-dimensional governing parameters.

The physics-based identification and modeling of individual noise sources is accomplished using a parameter identification technique applied to the separated rotor harmonic noise measurements. This technique is based on the inverse simulation of a relatively simple analytical model of helicopter noise generation, accepting the known independent governing parameters of the rotor harmonic noise sources as well as unknown dependent parameters which affect noise generation.

The inverse simulation is performed by evaluating the model repeatedly using different trial values for the dependent parameters and comparing the output of the simulated model to the measured data. The combination of dependent parameter values which results in the closest match between the simulated and measured data is then associated with the independent governing parameters that define the operating condition of the helicopter. By using a physics-based model, the effects of the primary factors of noise generation are exposed, making possible estimates of noise generated at other flight conditions than those measured. Parametric trends that are not captured by the simple analytical model are reflected in the variation of the dependant parameters with respect to the non-dimensional governing parameters. Due to the simplicity of the model, once the parameter identification technique has been completed, noise estimates can be made very quickly relative to direct simulation schemes.

$\mathrm{BVI}$ is one of the more challenging noise sources to accurately model, and for this reason is the focus of this paper. BVI is strongly affected by small changes to the geometry of the rotor wake with respect to the rotor tip-path plane. The distance between the vortex and blade during the interaction is termed the "miss distance", and has a strong effect on the magnitude of BVI noise. The "top view" geometry of the interaction controls how the intersection of the vortex and blade sweeps through the medium. (Figure 5) As the interaction geometry changes rapidly in both space and time the acoustic effect collects in phase, focusing the radiated impulse towards a particular direction. (Figure 6) Due to the speed and spatial extent of the underlying mechanism, BVI is poorly approximated by a compact source. 


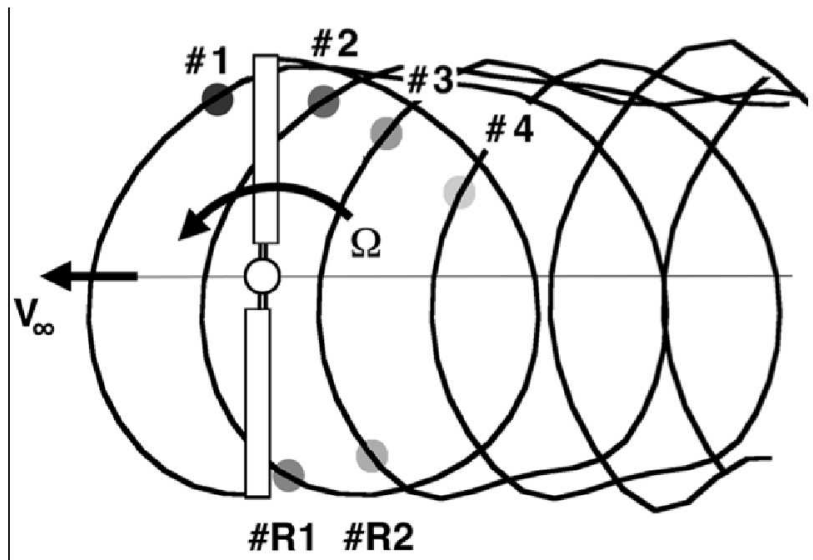

Figure 5: "Top view" BVI geometry.

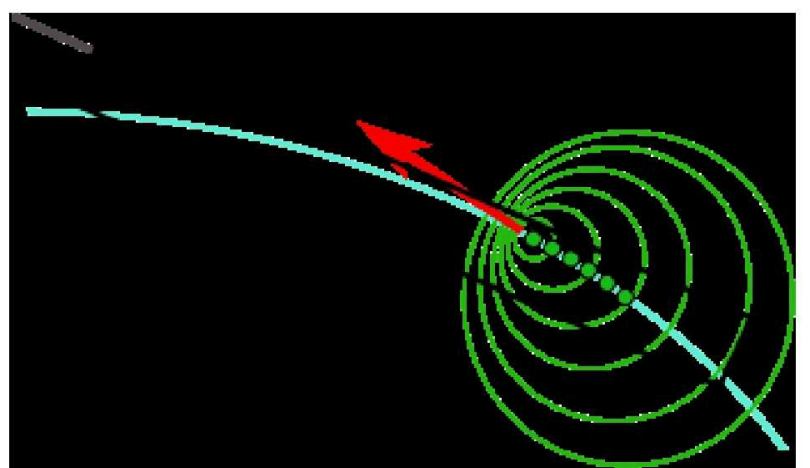

Figure 6: Illustration of the BVI phase collection process. The precise geometry of the interaction controls the directivity of radiated noise.

The physics-based model used in this paper is based on the Ffowcs Williams - Hawkings formulation of the acoustic analogy. This is solved using a multithreaded rigid blade on-surface Farassat Formulation 1A [7] routine developed for this purpose. Aerodynamic inputs to the acoustic model are calculated using a tunable prescribed wake model based on the Beddoes model [8] with Bhagwat-Leishman-type [9] vortex core growth (Figure 7 and Figure 8). (Similar in concept to the "Level C" modeling in [10].) The LeishmanBeddoes 2D incompressible indicial aerodynamics method [11] is used to account for unsteady aerodynamics effects. The simplicity of the modeling scheme allows for rapid run times enabling the parameter identification process to be completed in a reasonable amount of time - on the order of a few hours for a modern desktop computer. However, both the incompressible unsteady aerodynamics model and the on-surface acoustics model are limited in applicability to low to moderate advancing tip Mach numbers, and may not provide accurate predictions at higher flight speeds without correction.

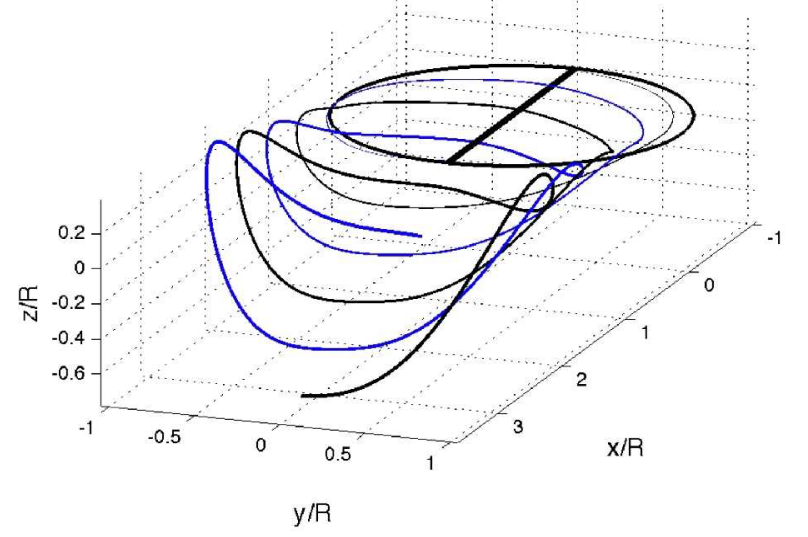

Figure 7: Geometry of the modified Beddoes wake model.

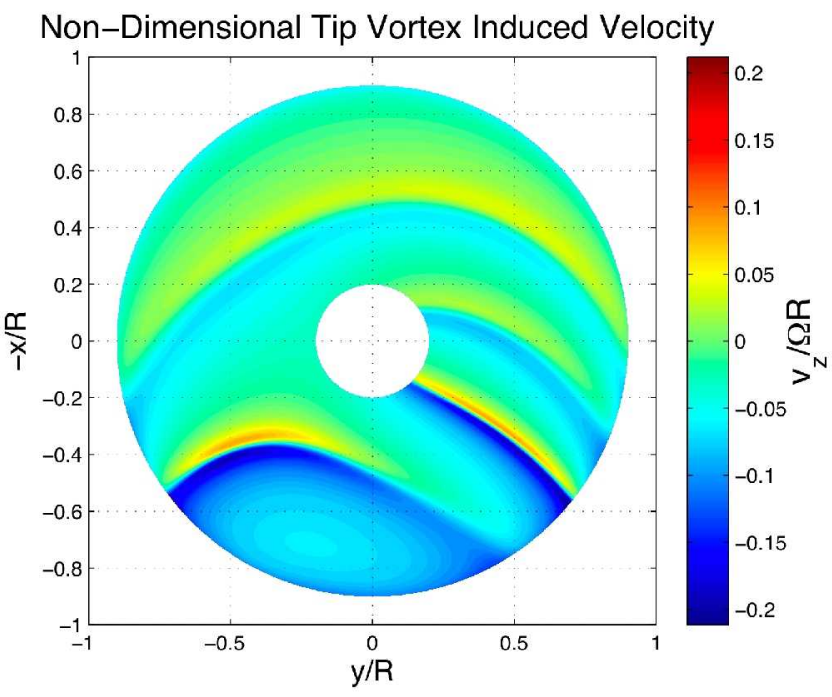

Figure 8: Distribution of velocities induced at the rotor blades by forward simulation of the wake model. The localized nature of BVI noise sources is apparent.

In addition to the known non-dimensional governing parameters of rotor harmonic noise, this model accepts several dependent parameters affecting noise generation for which there are no simple direct solutions. It is through the variation these dependent parameters that more complex aerodynamic effects are captured.

The inflow distribution across the rotor controls the "miss distance" between the vortex and blades, strongly influencing the strength of each type of BVI. The Beddoes wake model provides a means of relating the non-dimensional inflow and advance ratio to miss the distance. While the Beddoes model initially assumed only a longitudinal distribution of inflow, it has since been extended to include a lateral distribution, as well.[12] The model is further extended in this paper to include dependent modeling parameters allowing for a more complex variation of inflow distribution as an unknown function of the governing parameters $\mu, \lambda, C_{T}$, 
and $M_{A T}$. The expression used in the assumed model for the vertical displacement of vortex elements covered by the rotor disk is:

$$
\begin{aligned}
z=\mu_{z} \phi-\lambda_{i}\left[A\left(\cos \psi_{v}+\frac{\phi}{2 r_{v}}-\left|r_{v} \sin \psi_{v}\right|^{3}\right) \arctan \frac{\mu_{x}}{\mu_{z} \lambda_{i}}\right. \\
\left.+2 B \mu_{x} \sin \psi_{v}+C\right]_{\phi}
\end{aligned}
$$

where $A, B$, and $C$ are the dependent parameters controlling the longitudinal, lateral, and mean inflow, respectively.

The phasing of BVI is very important in determining the directivity and strength of the interactions. The phasing of BVI is related to the "top-view" geometry of the rotor wake. In addition to the governing nondimensional parameters of advance ratio $(\mu)$ and tip Mach number $\left(M_{A T}\right)$, the phasing of BVI events is controlled by the radial position of the tip vortices. Tip vortices roll-up somewhat inboard of the blade tips, and the diameter of the wake will proceed to contract over time. The parametric variations of these effects are included in the model by including two additional dependent modeling parameters:

$$
\begin{aligned}
& x=r_{v} \cos \psi_{v}+\mu_{x} \phi \\
& y=r_{v} \sin \psi_{v} \\
& r_{v}=r_{r}\left(D+(1-D) e^{-\lambda_{1} \phi}\right)
\end{aligned}
$$

where $r_{r}$ is the dependent parameter controlling initial tip vortex roll-up radius, $D$ is the dependent parameter controlling the rate of wake contraction, and $\lambda_{1}=0.145+27 C_{T}$, as suggested by Landgrebe.[14]

In addition to the wake geometry, there are other mechanisms that control the strength of BVI noise and are governed by the non-dimensional parameters. The initial vortex core size and growth rate control the diameter of viscous vortex core along the tip vortices, and therefore influence the "impulsiveness" of the BVI events and the resulting noise levels. The vortex core size is expressed in the model as:

$r_{c}=\sqrt{r_{0}^{2}+4 C_{v} \phi}$

where initial core size, $r_{0}$, and the vortex core growth rate, $C_{v}$, are both dependent parameters.

The strength of the tip vortex directly influences the strength of the BVI, and varies azimuthally. This is expressed in the assumed model using three dependent parameters:
$\Gamma=\Gamma_{0}\left(\gamma_{0}+\gamma_{1 S} \sin \psi_{v}+\gamma_{1 C} \cos \psi_{v}\right)$

where the nominal vortex strength $\Gamma_{0}$ is determined by the ideal vortex strength resulting from a constant triangular load distribution along the blade span:

$$
\Gamma_{0}=2 C_{T} \pi V_{t i p} R / b
$$

In addition to these parameters which primarily control the unsteady loads caused by BVI, lower harmonic loading sources are identified by specifying the harmonic coefficients of a linear distribution of thrust and drag loads along the span of the blades. The contribution of thickness noise to the main rotor harmonic time-history signal is calculated directly from the airfoil geometry and the known governing parameters. With the exception of high-speed impulsive noise, which only occurs at very high flight speeds, the contributions of all rotor harmonic noise sources are identified and modeled using this approach. The set of dependent parameters to be identified is then:

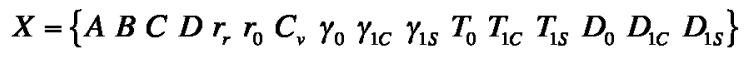

Determining the correct choice of dependent parameter values to input to the physics-based model for each set of known governing parameter values to match the measured noise signals is not a trivial task. Even when using a relatively low fidelity model, the computational cost of trying all feasible combinations of unknown parameter values in order to find the best match is impractically high. A better approach is to use optimization techniques to minimize the error between simulated and measured noise.

Formulating the parameter identification problem as an optimization problem requires developing an objective function which yields not only accurate match of acoustic radiation for any measured flight condition, but also limits the problem to a small set of feasible solutions which accurately represent the underlying physics of rotor noise generation. In order to incorporate as much physical information as possible, the phase of the radiated noise should not be ignored and therefore the error metric is calculated in the timedomain. This metric is formulated using the p-norm of the error between the simulated and measured noise as a function of time:

$$
\|e\|_{p}=\left(\int_{0}^{T}\left|p_{\text {meas }}^{\prime}(t)-p_{X}^{\prime}(t)\right|^{p} d t\right)^{\frac{1}{p}}
$$

The p-norm is an integrated measure of the difference between the two signals over the entire time period. 
(Figure 9) The choice of " $p$ " determines how strongly the peak values of error are weighted over the average value of error over the time period. Larger values of " $p$ " emphasize greater departures from the measured signal over the time period more heavily than smaller departures. For instance, for unity " $p$ ", the norm represents the average value of error across the period, whereas at the other extreme of " $p$ " equal to infinity, the value of the norm is determined only by the maximum error across the entire period.

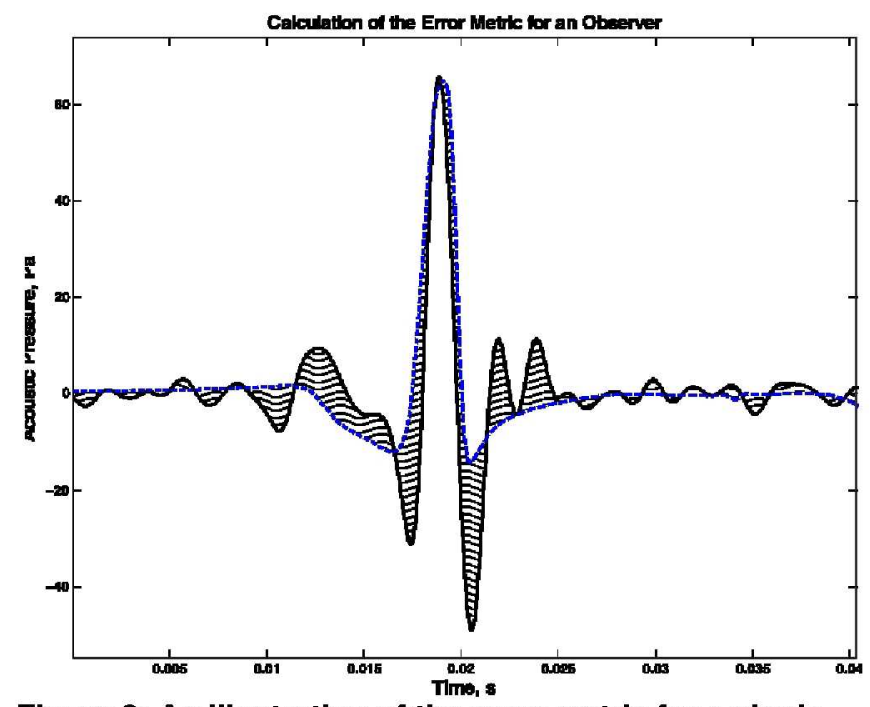

Figure 9: An illustration of the error metric for a single observer. Simulated BVI pulse (blue dashed), measured BVI pulse (black solid), integrated absolute error (hashed area).

Additionally, the solution to the pressure time history at any given observer location is non-unique - to improve the uniqueness of the solution and ensure that the governing physics are well modeled, multiple distinct observer locations must be considered in the formulation of the objective function. The overall objective function to be minimized is then a weighted sum of the error metrics calculated at each observer location:

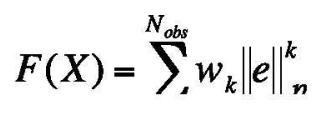

The weights, $w_{k}$, may be chosen so as to emphasize the accuracy of the fit in certain directions. For instance, out-of-plane observers may be more heavily weighted to better suited for community noise evaluation than long range detection.

Unfortunately, this objective function is unlikely to be purely convex. Numerous local minima are likely to exist over the parameter space, preventing conventional gradient-based optimization techniques from correctly identifying the set of parameters that minimize the objective function over the entire space of feasible parameters. While an enumeration would eventually find the best set of parameters, the time required to evaluate every possible set of feasible parameters to reasonable accuracy would be enormous. An efficient optimization algorithm able to cover a wide range of possible solutions must be utilized. Stochastic methods are now commonly applied to such problems; these methods include genetic algorithms, differential evolution, simulated annealing, and particle swarm optimization. In this paper, the particle swarm optimization approach was selected due to its more intuitive approach, rapid convergence on many types of physical problems, and adaptability to a wide range of problems.

Particle Swarm Optimization (PSO) [13] evaluates the objective function at a number of randomly seeded "particle" locations in the feasible solution space. The particles are assigned velocities dictating how they travel through the solution space. At fixed intervals, the objective function is again evaluated at each particle location, and a new velocity computed for each particle. The velocity of a particle during the next iteration $\left(v_{i}^{t+1}\right)$ is a randomly weighted combination of the current velocity direction $\left(v_{i}^{t}\right)$, the direction towards the previous best set of parameters for the particle ( $\left.X_{p b}^{i}\right)$, and the global best set of parameters discovered by any particle $\left(X_{g b}^{t}\right)$. (Figure 10) The PSO update algorithm can be expressed simply:

$$
\begin{aligned}
& v_{i}^{t+1}=a v_{i}^{t}+b_{1} \mathcal{R}_{1}^{t}\left(X_{p b}^{t}-X_{i}^{t}\right)+b_{2} \mathcal{R}_{2}^{t}\left(X_{g b}^{t}-X_{i}^{t}\right) \\
& X_{i}^{t+1}=X_{i}^{t}+v_{i}^{t}
\end{aligned}
$$

where $\mathcal{R}_{1}$ and $\mathcal{R}_{2}$ are random variables between 0 and $1, a$ is the "inertia weight" of the particle, and $b_{1}$ is the weight of the direction towards the historical best position of particle $i$, and $b_{2}$ the weight of the direction towards the best position of the entire swarm.

The stochastic nature of the algorithm encourages the particles to explore the entire parameter space while the inertia term prevents the solver from settling into local minima. The weights suggested by Trelea [15] are used in the method developed in this paper, however the number of particles in the swarm has been increased to 40 to ensure better initial coverage of the parameter space.

Once the PSO has converged on a set of dependent modeling parameters that minimize the objective function, the dependent parameters are associated with the non-dimensional governing parameters that 
define the measured flight condition. Supplying these parameters to the assumed analytical model will yield the same noise as was measured for the same flight conditions along the same directions. Additionally, the model may be used to predict noise at different directions or different flight conditions than measured.

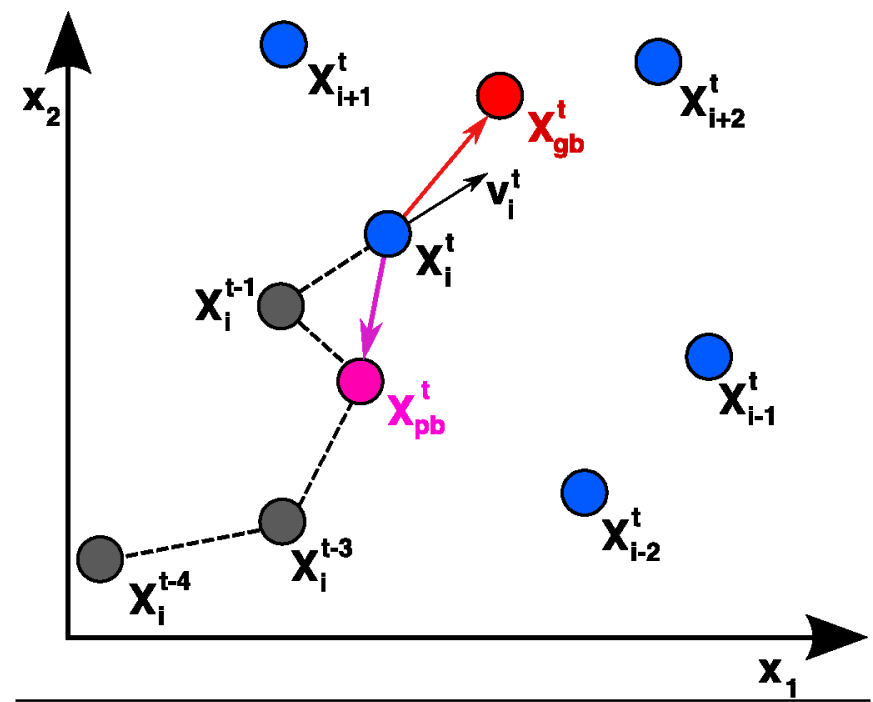

Figure 10: The Particle Swarm Optimization velocity update.

\section{Application to Wind Tunnel:}

The parameter identification method is first applied to acoustic measurements of several flight conditions produced from the test of the 2-bladed $1 / 7^{\text {th }}$ scale $\mathrm{AH}-1$ Operational Loads Survey (OLS) rotor in the GermanDutch Wind Tunnel (DNW).[4] The data from this wind tunnel test include high quality acoustic and blade pressure data. A wide range of operating conditions was measured, with controlled variation of the nondimensional governing parameters of rotor noise. Nine microphones located within the wind tunnel jet, labeled 2 through 10 in Figure 11, were included in the parameter identification process. Although these microphone locations are predominantly ahead of and towards the advancing side of the rotor, there is still a sufficient variation in directivity angles to allow a single "best fit" to be identified in all cases.

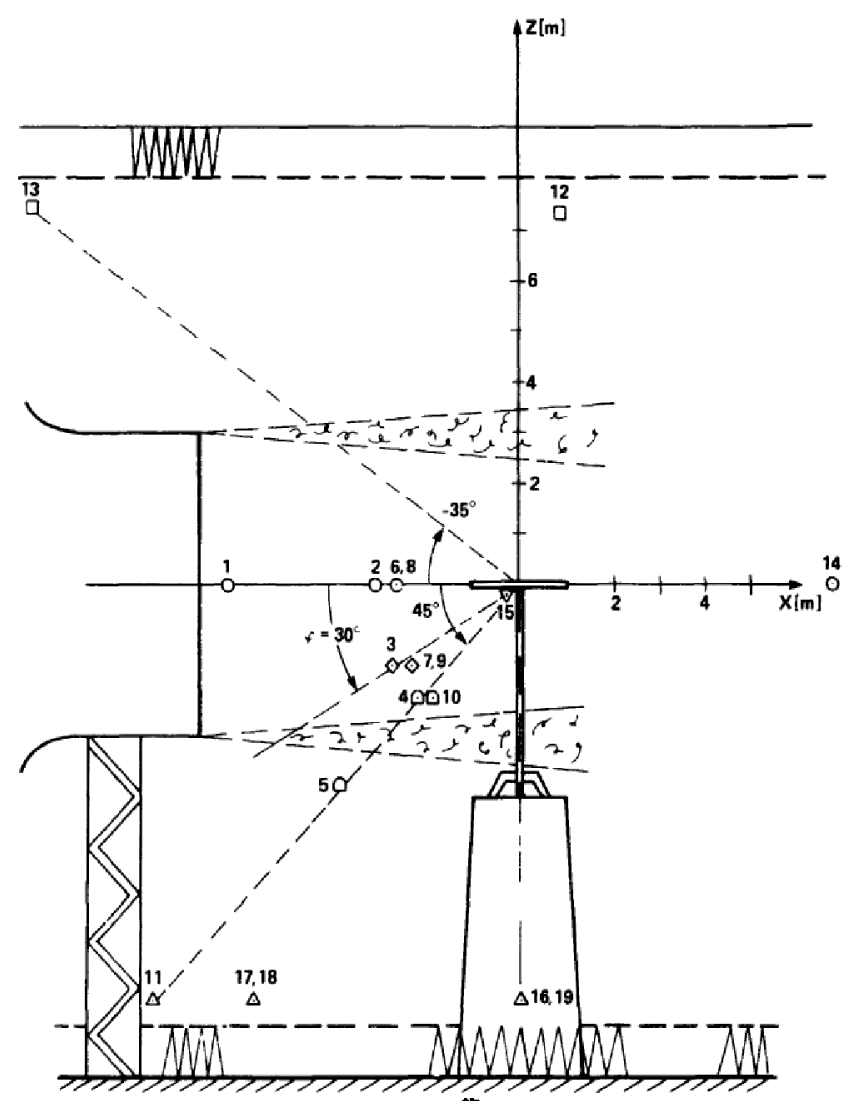

Figure 11: Side-view of the $1 \pi^{\text {th }}$ Scale OLS test microphone layout.[4]

In order to emphasize BVI impulses in the fitting process, the 8-norm is used to evaluate the time history error signals $(p=8$ in Eq. 11). For a uniform weighting of the error metrics at each observer location (i.e. all $w_{k}=1$ ), the fitting process produces pressure time-history signals within about $1 \mathrm{~dB}$ OASPL of measured values at most observer locations. At observer angles near the plane of the rotor, the fit is less accurate and fits to within about $3 \mathrm{~dB}$ OASPL. A representative selection of measured and modeled pressure time histories is shown for a BVI noise case in Figures 12-14. In general, in-plane BVI and thickness noise are not as well modeled. (Figure 14) 


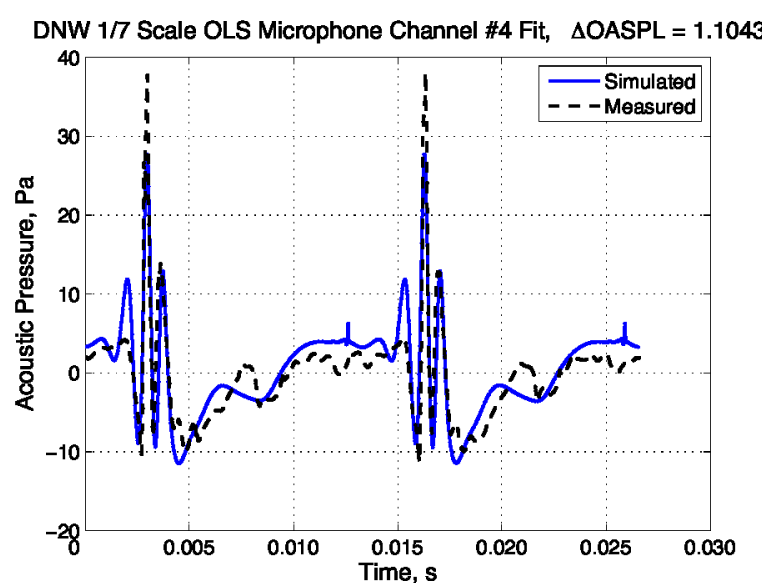

Figure 12: 45 degree elevation observer. BVI is well captured by the fitted model.

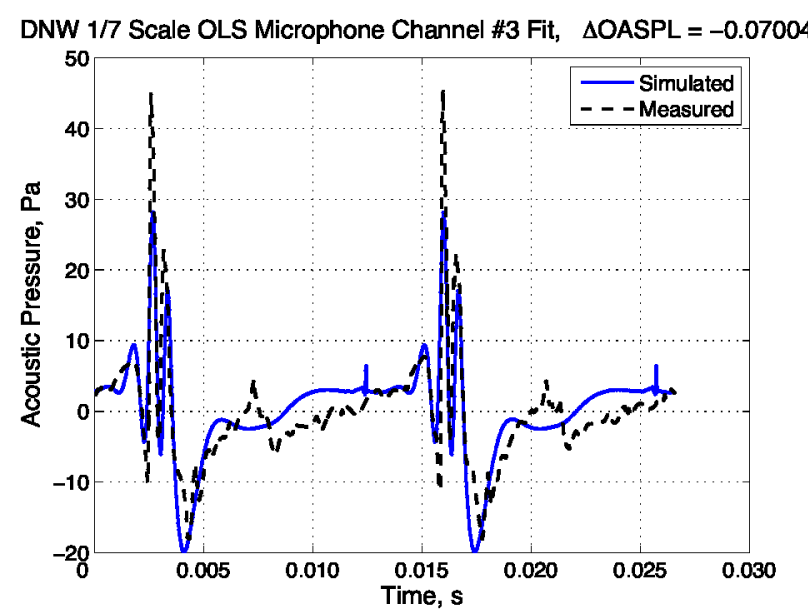

Figure 13: 35 degree elevation observer.

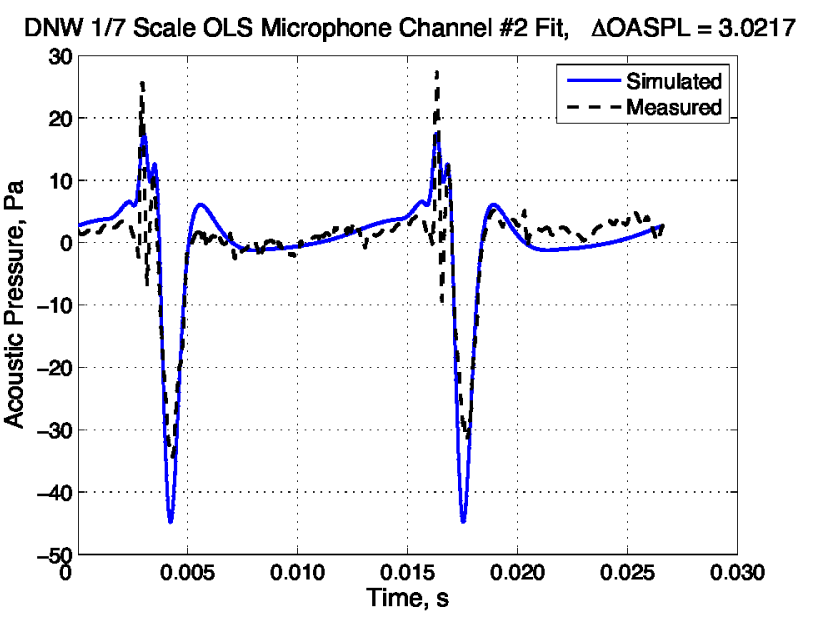

Figure 14: 0 degree elevation observer. In-plane BVI and thickness noise are underestimated by the fitted model.

Using the modeling parameters identified for a single test case of the model rotor, estimates are made for the noise radiated at other operating conditions, as defined by the non-dimensional governing parameters.
The identified flight condition is defined by the following parameter values:

\begin{tabular}{|l|l|}
\hline$\lambda$ & 0.0135 \\
\hline$C_{T}$ & 0.0054 \\
\hline$\mu$ & 0.164 \\
\hline$M_{A T}$ & 0.722 \\
\hline
\end{tabular}

First, thrust coefficient is varied while keeping the values of the other three non-dimensional governing parameters constant. An increase in thrust coefficient yields an increase in the circulation strength of the tip vortices, generating larger changes in section angle of attack during BVI and hence larger amplitude BVI noise impulses. The estimated variation is compared with previous measurements for a microphone located directly in front of and 45-degrees below the model rotor tip-path plane. [4] The results are plotted in Figure 15.

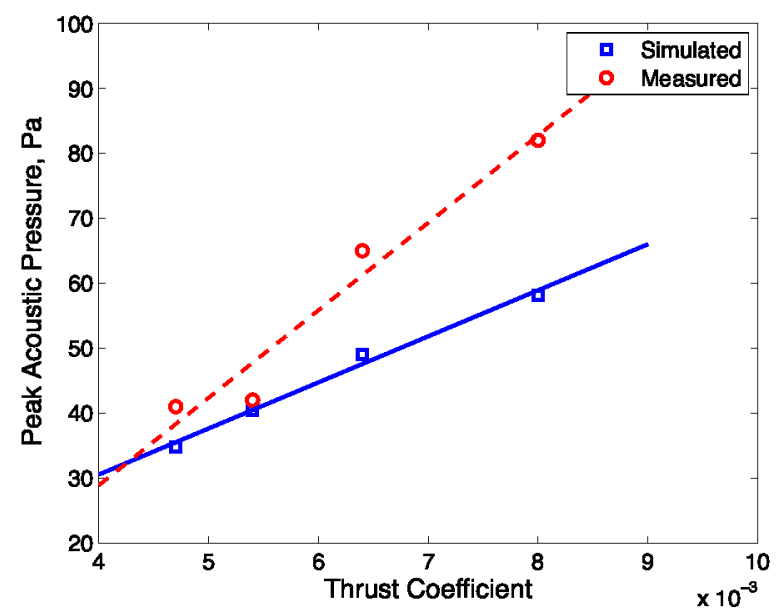

Figure 15: Peak acoustic pressure versus thrust coefficient for the $\mathbf{4 5}$ degree elevation observer at $\boldsymbol{M}_{A T}=$ 0.722. Time history for $C_{T}=\mathbf{0 . 0 0 5 4}$ shown in Figure 12 .

In both cases, the variation of BVI impulse amplitude varies linearly with thrust coefficient, however the sensitivity of the fitted model to thrust variation is less than was measured. The experimental case used in fitting the model exhibits a lower peak pressure than the linear trend would suggest, leading the model to underpredict noise levels at other thrust coefficients. More complex variation in noise levels can only be captured by the assumed model through inclusion of dependent modeling parameter information for models fitted for other conditions. In order to simulate this process with the available data, the pressure time history signals from fitted case $\left(C_{T}=0.0054\right)$ were scaled-up in amplitude to match the peak level reported at the 45-degree microphone for the highest thrust case $\left(C_{T}=0.0080\right)$. The parameter identification process was run on the modified data using the higher thrust coefficient, generating an additional set of 
dependent modeling parameters. Interpolation of these dependent parameters with respect to variations in the governing parameters allows for a muchimproved model of the effect of parametric variations on noise radiation. (Figure 16) Including more measurements in the model building process allows for even improved interpolation of the dependent modeling parameters, further increasing the accuracy of noise estimates. (Figure 17)

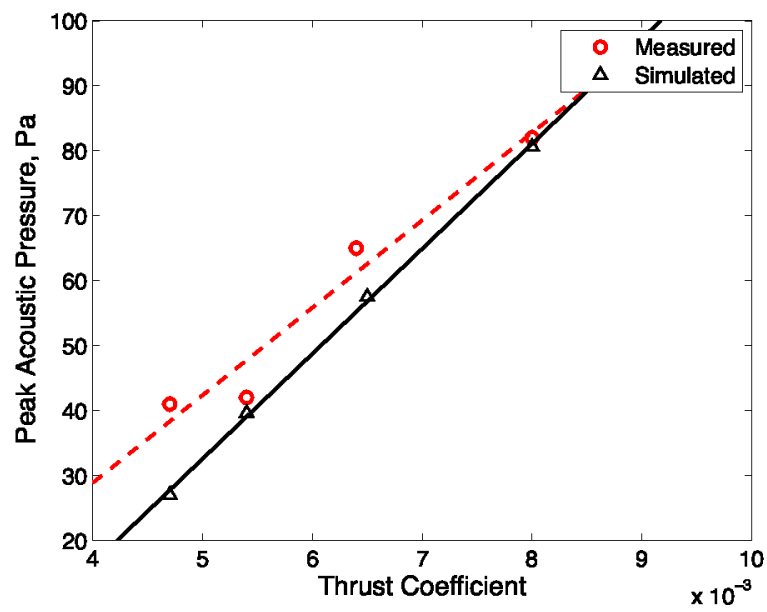

Figure 16: Peak acoustic pressure versus thrust for model generated using measured data at $C_{T}=0.0054$ and estimated data at $C_{T}=0.0080 .\left(M_{A T}=0.722\right)$

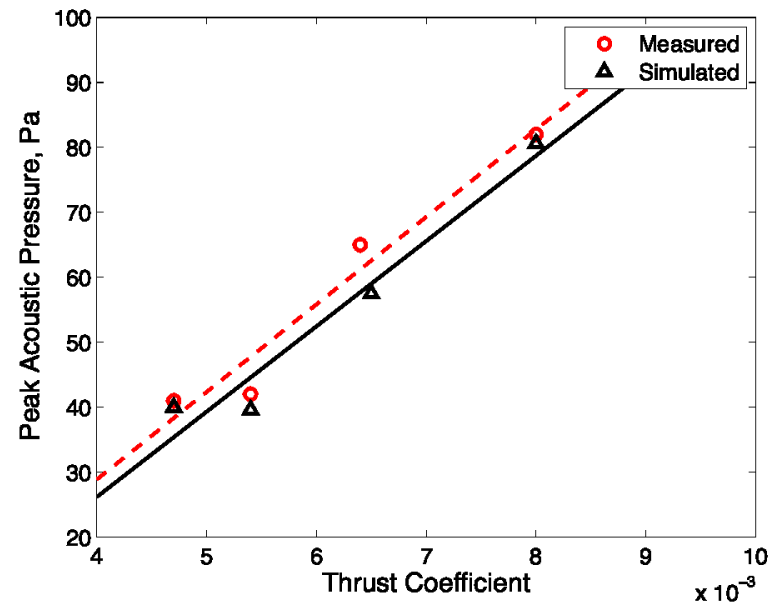

Figure 17: Peak acoustic pressure versus thrust for model generated using measured data at $C_{T}=0.0054$ and estimated data at $C_{T}=0.0047$ and $C_{T}=0.0080$. $\left(M_{A T}=0.722\right)$

The parametric variation of tip Mach number was also estimated using the initial fitted model and compared to measured data in Figure 18.

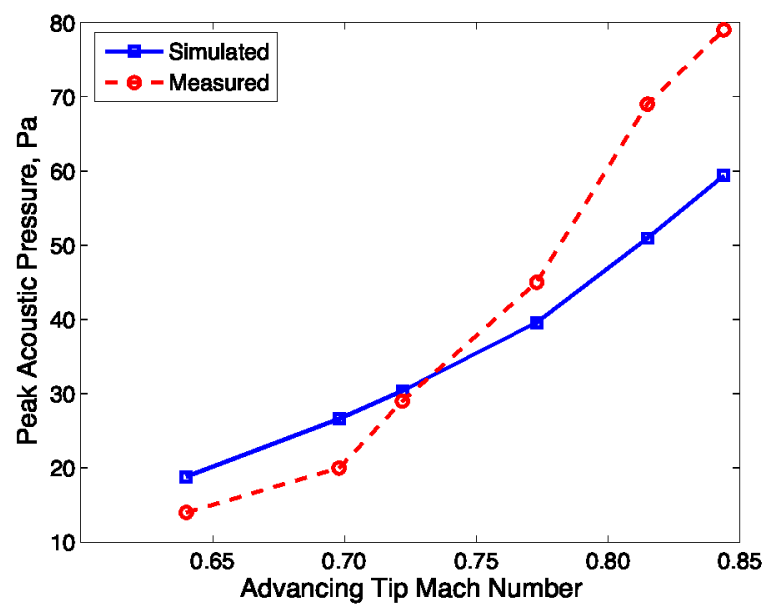

Figure 18: Peak acoustic pressure versus advancing tip Mach number for the $\mathbf{4 5}$ degree elevation observer for $C_{T}$ $=0.0054$. Time history for $M_{A T}=0.722$ shown in Figure 12.

Once again, the sensitivity of the fitted analytical model to advancing tip Mach number is less than that of the measured data. The low-order assumed model does not model the effects of compressibility on the unsteady airloads, leading to an underprediction of noise levels at high advancing tip Mach number. As for the case of thrust variation, the effects of large variations in non-dimensional governing parameters can be captured through a known variation of the dependent modeling parameters using measured data. (Figure 19)

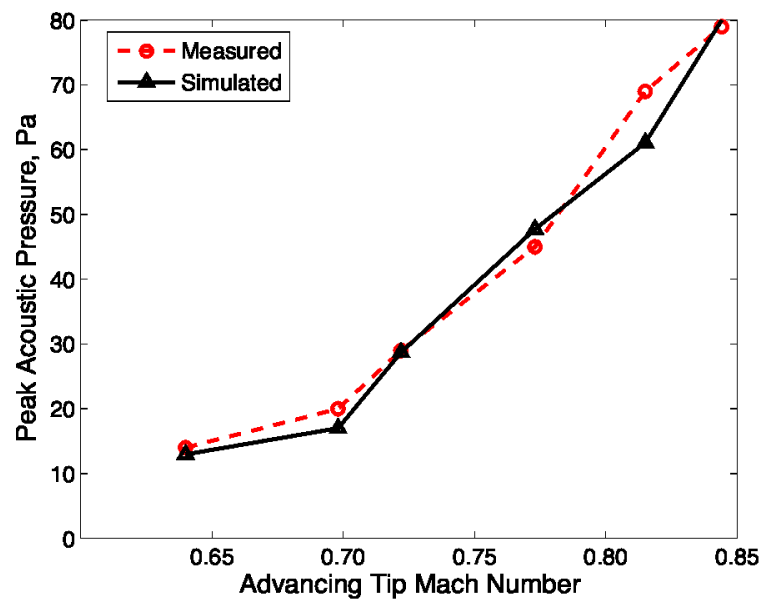

Figure 19: Peak acoustic pressure versus advancing tip Mach number using measured data at $M_{A T}=0.722$ and estimated data at $M_{A T}=0.640$ and $0.844 .\left(C_{T}=0.0054\right)$ 
By adding information from two additional data points, reasonably good agreement with measured data is achieved across a wide range of advancing tip Mach number. The fitted analytical model is also used to estimate noise levels at observer locations not directly measured by the acoustic array.

Figure 20 shows the Lambert projection of an acoustic hemisphere located 30 feet $(\sim 10 \mathrm{R})$ from the hub of the model rotor. Noise levels are shown using the BVISPL metric, defined in this paper as the overall sound pressure level of noise contained in the $6^{\text {th }}$ through $40^{\text {th }}$ harmonics of the blade passage frequency of the rotor. For this flight condition, noise is radiated ahead of and towards the advancing side of the rotor. By holding hover tip Mach number constant, and varying the advance ratio, estimates can be made for noise directivity and levels with variations in flight speed. The directivity variation with advance ratio is shown in Figures 21-23.

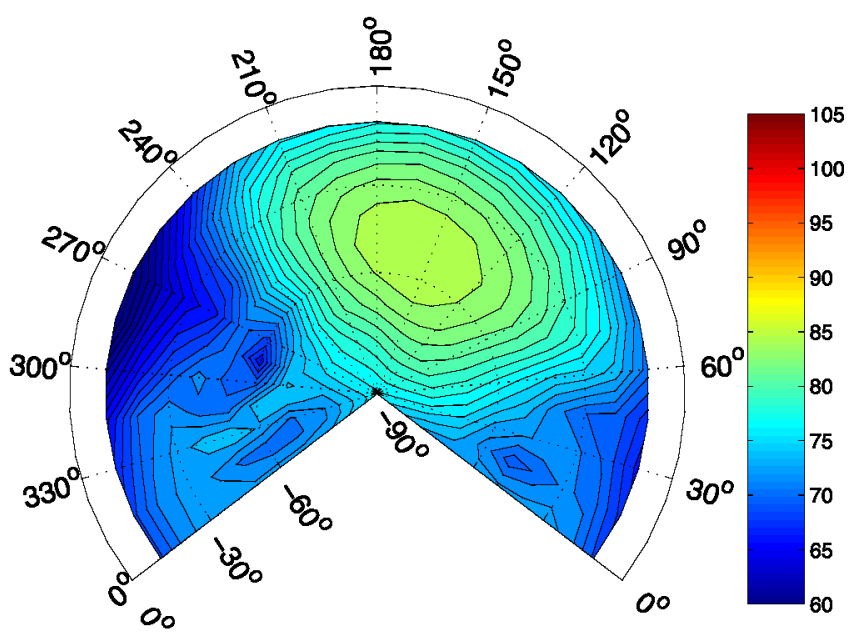

Figure 20: Lambert projection of the acoustic hemisphere produced using the fitted model. Noise contours are in BVISPL.

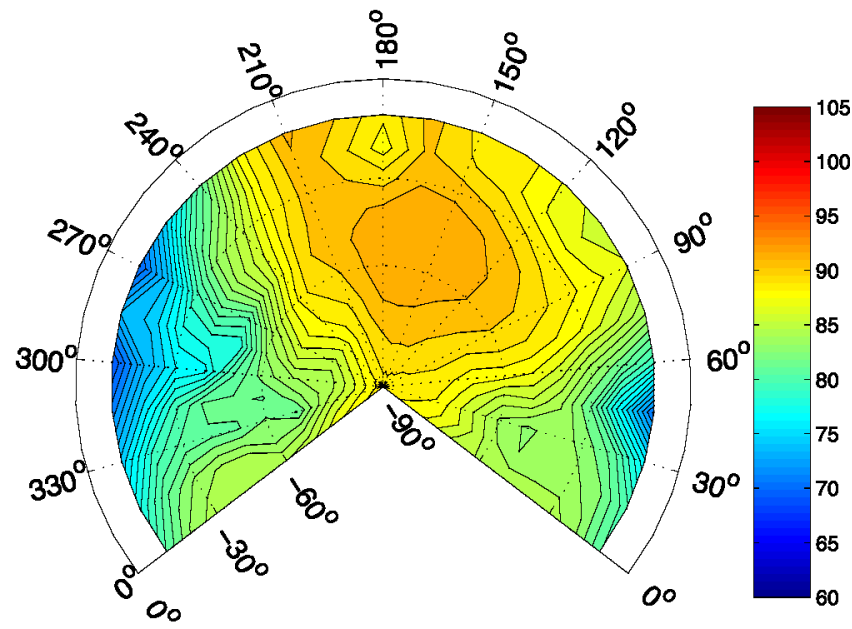

Figure 21: Estimated BVISPL hemisphere for $\mu=0.194$

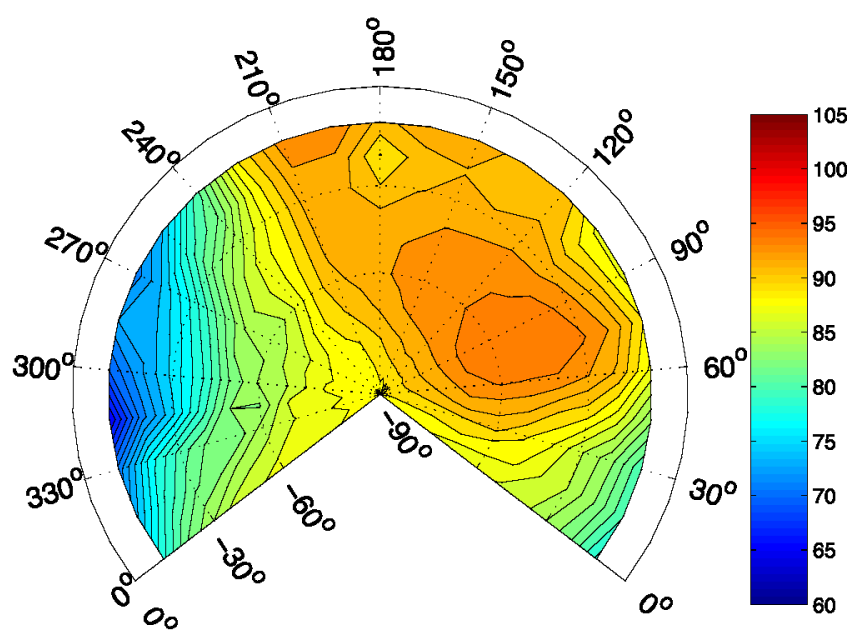

Figure 22: Estimated BVISPL hemisphere for $\boldsymbol{\mu}=\mathbf{0 . 2 2 4}$

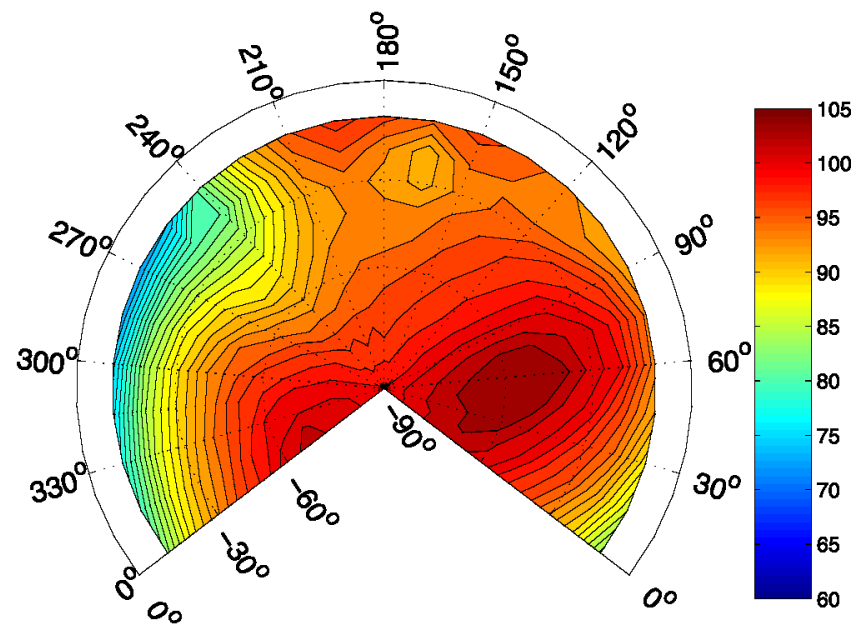

Figure 23: Estimated BVISPL hemisphere for $\mu=0.270$

As expected, as forward speed increases, advancing tip Mach number increases and noise levels increase. The predicted directivity of the radiated BVI noise "hotspot" tends to shift more out-of-plane and towards the advancing side with increasing advance ratio. 


\section{Application to Flight Test:}

The parameter identification approach is also applied to measured ground noise data from the flight test of a Bell 206B3 helicopter at Moffett Field, CA, in 2006.[5] Ground noise data was collected from a linear array of eight ground board microphones for a variety of steady and maneuvering flight conditions. In addition, the helicopter was instrumented with a unique inflight measurement array. The parameter identification technique is applied to this dataset to demonstrate how analytical models that relate noise to non-dimensional governing parameters may be built for full-scale helicopters. Such models are suitable for ground noise contour generation under a wide range of flight conditions. Ground noise contours for a diverse range of atmospheric and flight conditions are often required for effective mission planning and community noise abatement, yet measured ground noise data are generally available over only a limited set of operating conditions, primarily due to the significant cost of acquiring such data. Analytical models provide a means to generalize a limited set of measured data so that it is applicable over a wider range of conditions. In addition, the relatively simple analytical models resulting from this technique can be run in real time, which makes them suitable for inflight noise estimation.

As described in the previous section, the ground-based acoustic measurements are transformed to a frame analogous to wind tunnel measurements. The wavelet transform is then used to identify individual rotor revolutions from the acoustic signal. The main rotor pressure time history signals are extracted using a periodic averaging process for a number of observer directions. More rotor periods may be included in the averaging process for in-plane observers than out-ofplane observers; therefore, the more in-plane data is a better representation of the main rotor time history signal. In addition, the dominant noise sources of the rotor do not strongly radiate directly underneath the helicopter. For these reasons, the distribution of "virtual inflight observers" included in the analysis is biased away from the underside of the helicopter and towards the dominant radiation directions. The measured signals transformed to these observers are the inputs to the parameter identification process. Due to the increased variability in measured flight test data, the parameter identification process does not match the measured data as closely as for the wind tunnel case. However, the fit is within $2.5 \mathrm{~dB}$ OASPL for all observer locations - within the typical variations between repeated measurements of the same operating condition in flight test data. A representative fit of the pressure time histories is shown in Figure 24.

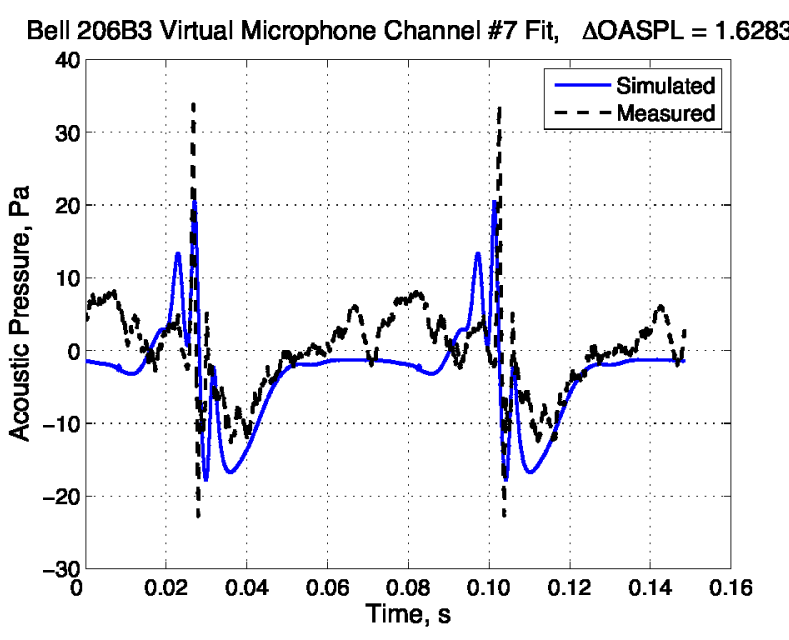

Figure 24: Time history signal simulated and measured for inflight observer at $\mathbf{3 0}$ degrees elevation, 150 degree azimuth. Bell 206B3 at 7.5-degree descent, 60 kts airspeed.

The process is applied to data for several steady descending flight conditions at 60 kts airspeed with approximately the same gross weight and rotor RPM for each case - in effect, a parametric variation of nondimensional inflow greatly affecting BVI noise levels.

Hemispheres using measured data for the main rotor harmonic noise in the BVISPL frequency range are shown for the -6 and -7.5 degree flight path angle cases in Figure 25 and Figure 26, respectively. Due to the high drag of the helicopter in the experimental configuration, $\mathrm{BVI}$ is encountered at steeper descent angles than the "clean" configuration. In the -6 degree case, a strong advancing side BVI is present. In the 7.5 degree case, the miss distance between the rotor blades and vortices decreases further, and the magnitude of this BVI is increased; in addition, there is evidence of significant retreating side BVI noise directed beneath the helicopter. This flight condition represents the highest BVI noise levels measured in steady descending flight during the test program. The microphone measurement locations covered by the "virtual inflight array" are shown by the dotted lines on each hemisphere - estimated noise levels between these points are interpolated. 


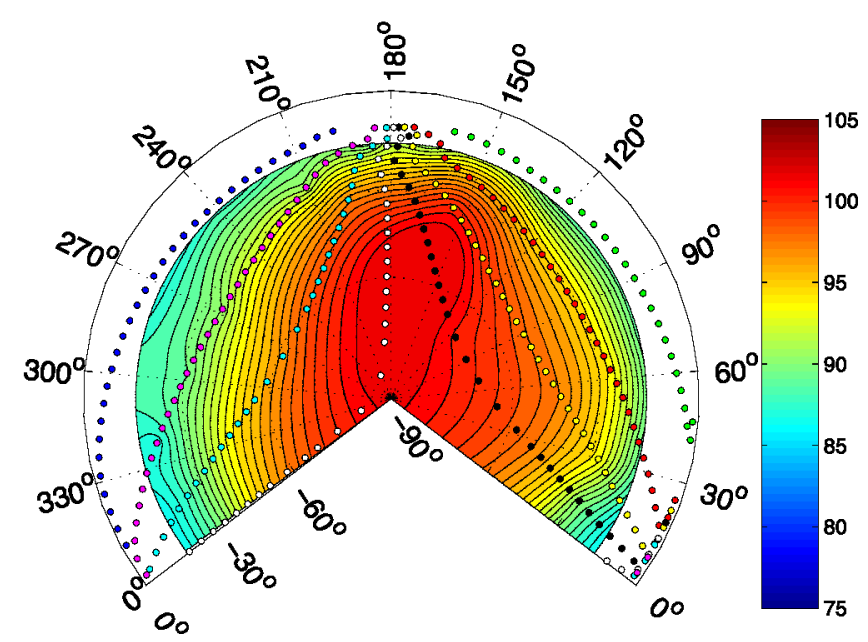

Figure 25: Main rotor averaged BVISPL hemisphere for -6 degree descent. $(\lambda=0.00659)$ Max BVISPL $=99.7$

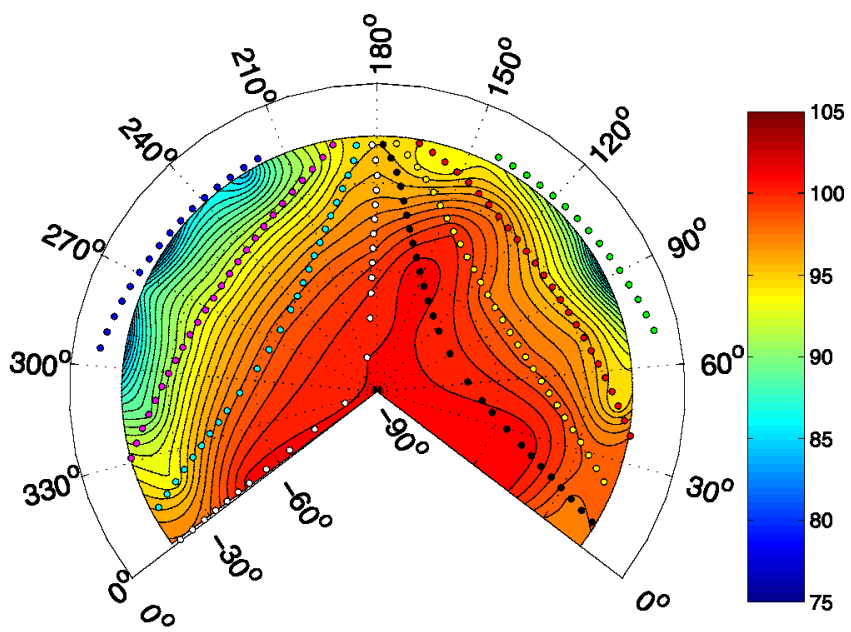

Figure 26: Main rotor averaged BVISPL hemisphere for -7.5 degree flight path angle. $(\lambda=0.00293)$ Max BVISPL $=101.4$

Figure 27 and Figure 28 show the simulated hemispheres generated from the assumed analytical model using the dependent parameters identified from the measured data for each case. The same general directivity trends and levels are observed in the modeled hemispheres as for the measured data, but the BVI directivity contours are much more distinct in the hemispheres generated by the analytical models. This is because the measured data must be time averaged in order to compensate for variability in the flight condition during the measurement process, and then interpolated over the surface of the hemisphere in order to compensate for the relatively sparse distribution of measurements. This results in reduced accuracy on the underside of the hemisphere, where the measurement geometry changes more quickly in time and fewer periodic averages may be taken over a given angle. Conversely, the fitted analytical models estimate noise levels in regions not actually measured based on physical modeling of the noise sources. As was shown in the wind tunnel application, more

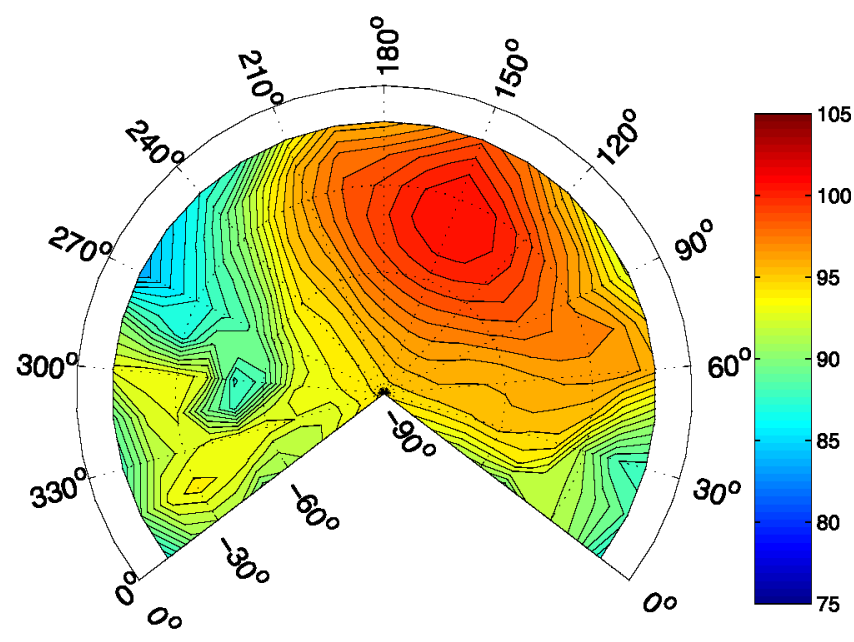

Figure 27: Simulated BVISPL hemisphere for -6 degree flight path angle. $(\lambda=0.00659)$ Max BVISPL = 99.5

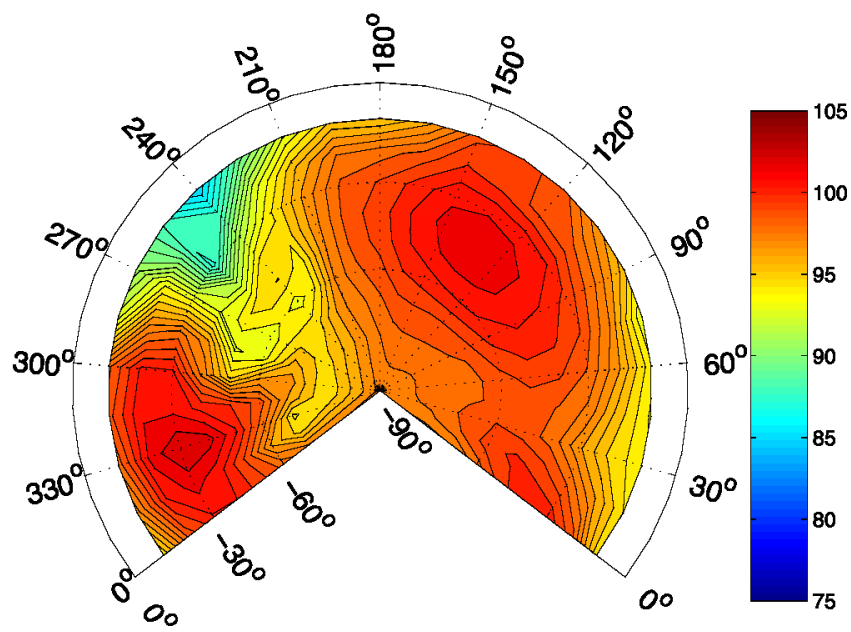

Figure 28: Simulated BVISPL hemisphere for $\mathbf{- 7 . 5}$ degree flight path angle. $(\lambda=0.00293)$ Max $B V I S P L=101.5$

accurate estimates of the noise generated by flight conditions not measured may also be made by interpolating the dependent modeling parameters discovered by the parameter identification process with respect to the non-dimensional governing parameters that classify each flight condition. For example, the noise levels observed at the -7.5 degree flight condition may be estimated using the modeling parameters determined from the -3 degree (Figure 29) and -9 degree (Figure 30 ) flight conditions by a linear interpolation of the dependent parameters based on the change in non-dimensional inflow. 


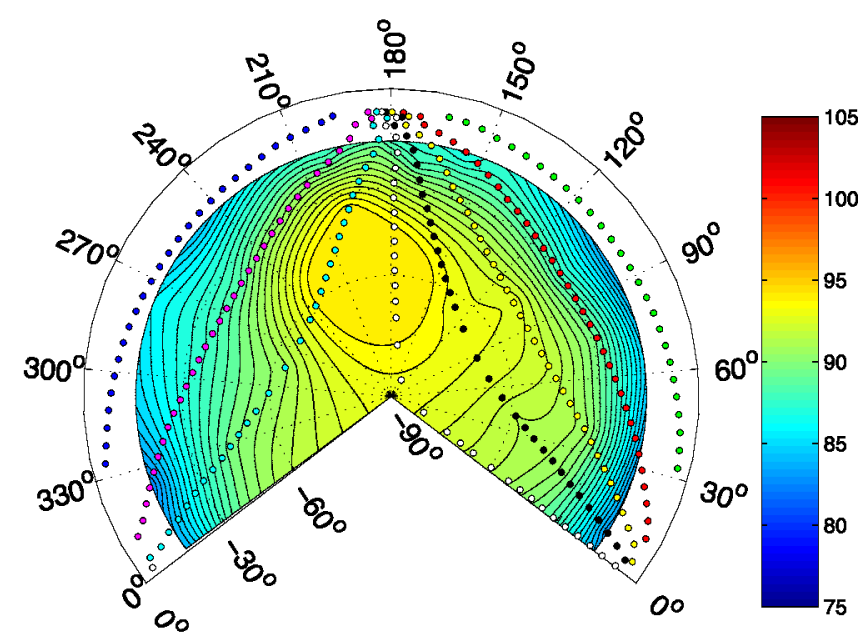

Figure 29: -3 degree BVISPL hemisphere. $(\lambda=0.01386)$

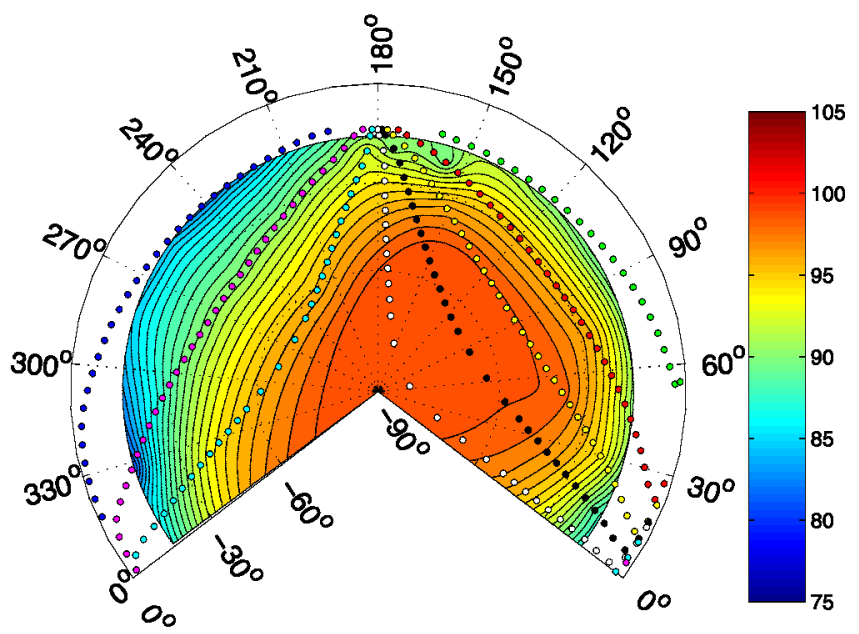

Figure 30: -9 degree BVISPL hemisphere. $(\lambda=-0.00074)$

Using the standard approach of linearly interpolating the SPL metric on the surface of the hemisphere based on the change in flight path angle (e.g. RNM [1]) results in the hemisphere shown in Figure 31. It is readily apparent that the estimated directivity and levels produced by this "data-driven" interpolation approach have little relation to the BVISPL contours measured for this condition, as shown in Figure 26. The BVI hotspot is directed farther towards the retreating side, and the maximum BVISPL on the hemisphere is $4 \mathrm{~dB}$ lower than measured. Without a physics-based model of $\mathrm{BVI}$, the simple interpolation approach is unable to predict the occurrence of BVI noise along directivity angles where it did not occur in the measured data.

Figure 32 shows the estimated BVISPL hemisphere for the same condition using an interpolation of the modeling parameters input to the assumed analytical

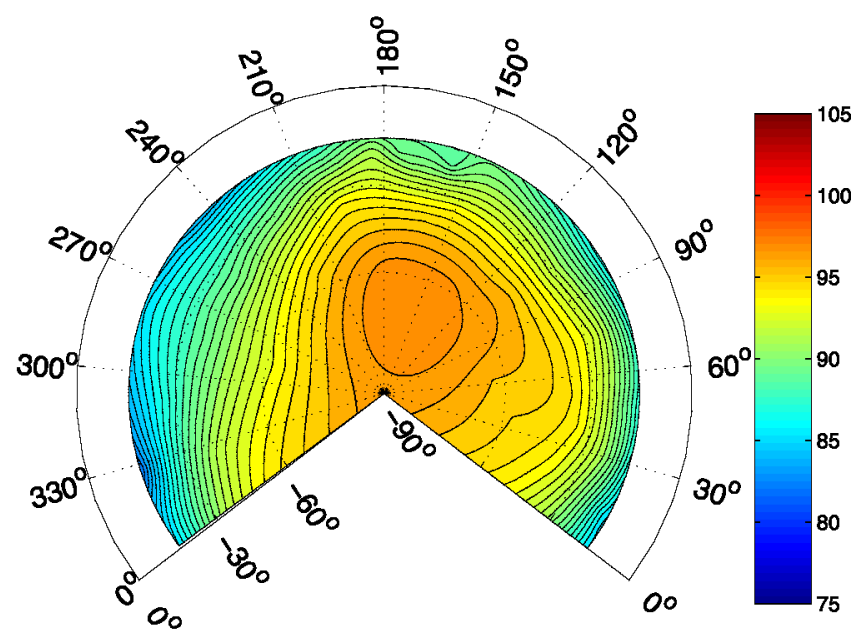

Figure 31: Estimated BVISPL hemisphere for $\mathbf{- 7 . 5}$ degree flight path angle $(\lambda=0.00293)$ using "data-driven" linear interpolation of noise contours. Max BVISPL = 97.4

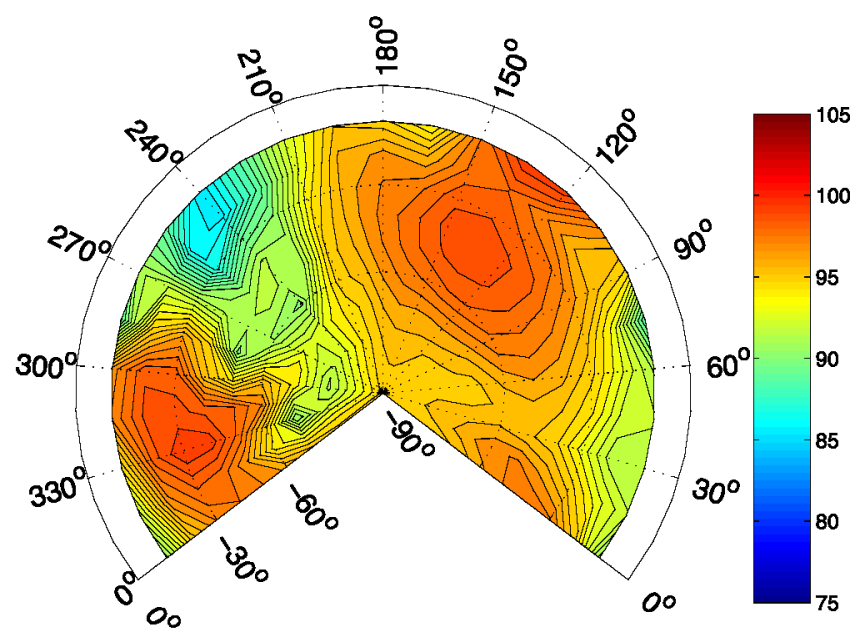

Figure 32: Estimated BVISPL hemisphere for $\mathbf{- 7 . 5}$ degree flight path angle $(\lambda=0.00293)$ based on -3 and -9 degree hemispheres. $(\lambda=0.01386$ and -0.00074$)$

Max BVISPL $=100.7$

model. By using a physics-based model, directivity patterns of BVI noise can be estimated which are distinct from those observed in the measured data. The noise levels and directivity agree much more closely with the measured data. BVISPL in the BVI "hotspots" are about $1 \mathrm{~dB}$ lower than those measured. The analytical model also produces a more distinct directivity pattern than the hemisphere produced directly from measurements, since the data do not need to be interpolated from sparse data across the surface of the hemisphere. The parameter identification scheme more accurately predicts noise levels and trends than simple data-driven interpolation, even for large changes in the governing parameters. 


\section{Conclusions:}

In this paper, a semi-empirical method of characterizing and modeling helicopter rotor harmonic noise sources in terms of their non-dimensional governing parameters has been developed using physics-based principles. The parameter identification method developed in this paper allows the contributions of individual rotor noise generation mechanisms to the overall measured noise to be determined. Although applicable to all rotor harmonic noise sources, this paper focuses on the particular application of the parameter identification method to BVI.

The parameter identification method has been successfully applied to data from the wind tunnel test of the $1 / 7^{\text {th }}$ scale OLS rotor. The model developed by this method closely matches the measured variation of BVI noise levels at all measurement locations over the normal operating range of tip-path-plane angles. Prediction of peak noise levels at other measured conditions was not as successful until additional data capturing variations in noise levels due to variations in the governing parameters were included in the parameter identification process. In particular, when the measured data set included conditions at other thrust coefficient levels and advancing tip Mach numbers, the comparison between the model and the measured data was good.

The parameter identification method was also applied to ground noise measurements of the Bell 206B3 helicopter. The analytical models resulting from the parameter identification method were able to estimate BVI noise levels in directions that were difficult to capture using the authors' previous rotor harmonic noise measurement technique [6]. The method also facilitated the accurate estimation of BVI noise at flight conditions not included in the set of measured data, even across wide variations in the non-dimensional governing parameters where standard "data-driven" interpolation of the measured noise levels failed.

The idea of combining the use of measured acoustic data set with another measured acoustic data set that was taken on the same type of rotor system but in a different test program is a new, powerful way of developing a higher fidelity noise estimation model. By using the physics-based parameter identification approach in this way, it should be possible to combine acoustic data from wind tunnel and flight testing to build robust and accurate rotorcraft noise source models.
The new method developed in this paper will facilitate the development of new source noise models for rotorcraft that can be extended to other operating conditions with a minimum of flight testing and computational expense. The new acoustic modeling method introduced in this paper should improve the accuracy of source noise prediction and aid in the development of mission and land use planning tools.

\section{References:}

[1] Conner, D.A., and Page, J., "A Tool for Low Noise Procedures Design and Community Noise Impact Assessment: the Rotorcraft Noise Model (RNM)," presented at the AHS International Meeting on "Advanced Rotorcraft Technology and Life Saving Activities," Heli Japan, Japan, November 2002.

[2] Browne, R.W., Munt, R.M., Simpson, C.R., and Williams, T., "Prediction of Helicopter Noise Contours for Land Use Planning," presented at the $10^{\text {th }}$ AIAA/CEAS Aeroacoustics Conference, Manchester, Great Britain, May 2004.

[3] Guntzer, F. Spiegel, P., and Lummer, M. "Genetic Optimizations of EC-135 Noise Abatement Flight Procedures using an Aeroacoustic Database," presented at the $35^{\text {th }}$ European Rotorcraft Forum, Hamburg, Germany, September 2009.

[4] Boxwell, D.A., Schmitz, F.H., Splettstoesser, W.R., and Schultz, K.J., "Helicopter Model Rotor-Blade Vortex Interaction Impulsive Noise: Scalability and Parametric Variations," Journal of the American Helicopter Society, Vol 32, (1), January 1987.

[5] Schmitz, F.H., Greenwood E., Sickenberger, R., Gopalan, G., Sim, B.W., Conner, D.A., Moralez, E., and Decker, W., "Measurements and Characterization of Helicopter Noise in SteadyState and Maneuvering Flight," presented at the American Helicopter Society $63^{\text {rd }}$ Annual Forum, Virginia Beach, VA, May 2007.

[6] Greenwood, E., and Schmitz, F.H., "Separation of Main and Tail Rotor Noise from Ground-Based Acoustic Measurements using Time-Domain DeDopplerization," presented at the $35^{\text {th }}$ European Rotorcraft Forum, Hamburg, Germany, September 2009.

[7] Brentner, K.S., "Prediction of Helicopter Rotor Discrete Frequency Noise," NASA TM-87721, October 1986.

[8] Beddoes, T.S., "A Wake Model for High Resolution Airloads," presented at the $2^{\text {nd }}$ International Conference on Rotorcraft Basic Research, Triangle Park, NC, February 1985.

[9] Bhagwat, M.J., and Leishman, J.G., "Generalized Viscous Vortex Model for Application to FreeVortex Wake and Aeroacoustic Calculations," presented at the American Helicopter Society $58^{\text {th }}$ Annual Forum, Montreal, Canada, June 2002. 
[10] Schmitz, F.H., and Sim, B.W., "Acoustic Phasing, Directivity, and Amplification Effects of Single Rotor Helicopter Blade-Vortex Interaction," Journal of the American Helicopter Society, Vol. 46, (4), October 2001, pp. 273-282.

[11] Leishman, J.G. "Validation of Approximate Indicial Aerodynamics Functions for Two-Dimensional Subsonic Flow," Journal of Aircraft, Vol. 75, (10), October 1988.

[12] van der Wall, B. "The Effect of HHC on the Vortex Convection in the Wake of a Helicopter Rotor," Aerospace Science \& Technology, 4, 2000, pp. 321-336.

[13]Eberhart, R.C, Kennedy, J. "A new optimizer using particle swarm theory," presented at the Institute of Electronics and Electrical Engineers Conference on Neural Networks, Perth, Australia, 1995.

[14] Landgrebe, A.J. "The wake geometry of a hovering helicopter rotor and its influence on rotor performance," Journal of the American Helicopter Society, Vol. 17, (4), October 1972.

[15] Trelea, I.C. "The particle swarm optimization algorithm: convergence analysis and parameter selection," Information Processing Letters, Vol. 85, (6), 2003, pp. 317-325. 